\title{
Gaussian limits for discrepancies I. Asymptotic results
}

\author{
André van Hameren ${ }^{\mathrm{a}, 1}$, Ronald Kleiss ${ }^{\mathrm{a}, 2}$, Jiri Hoogland ${ }^{\mathrm{b}, 3}$ \\ a University of Nijmegen, Nijmegen. The Netherlands \\ b CWI, Amsterdam. The Netherlands
}

Received I September 1997

\begin{abstract}
We consider the problem of finding, for a given quadratic measure of non-uniformity of a set of $N$ points (such as $L_{2}$ star-discrepancy or diaphony), the asymptotic distribution of this discrepancy for truly random points in the limit $N \rightarrow \infty$. We then examine the circumstances under which this distribution approaches a normal distribution. For large classes of non-unitormity measures, a 'Central Limit Theorem' can be derived. (C) 1997 Published by Elsevier Science B.V.
\end{abstract}

\section{Introduction}

In the field of numerical integration, there are two aspects of the general problem which bear on the accuracy of the numerical result. The first is, of course, the behaviour of the integrand: typically, wildly fluctuating functions are integrated with less accuracy than relatively smooth ones, for the same number of integration points. The second one is the distribution of the set of points at which one evaluates the integrand. It stands to reason that, if one has no a priori knowledge of the integrand, a set of points that is fairly uniformly distributed may be expected to do better than one in which many points cluster together. It is therefore useful to quantify and study the notion of 'uniformity of point sets', and this has been the topic of a great number of publications $[1,2]$. The most important of such notions are those of the star-discrepancy and $L_{2}$ stardiscrepancy, and more recently other measures of non-uniformity that go under the name of diaphony have been introduced as well [3]. In this paper, we shall call all such measures 'discrepancies'.

As has been shown in Ref. [4,5], the use of a particular discrepancy in assessing the uniformity of a given point set implies that one has some notion of the generic behaviour of the integrand: it is tacitly assumed that the integrands to be attacked belong to some class of functions. The particular discrepancy is then recognized as the average-case complexity of the integration problem over that function class $[6,7]$.

\footnotetext{
${ }^{1}$ E-mail: andrevh@sci.kun.nl

2 E-mail: kleiss@sci.kun.nl

${ }^{3}$ E-mail: jiri@cwi.nl
} 
While the Monte Carlo method, in which the integration points are chosen at random, has long been recognized as a robust and useful way of evaluating multivariate integrals, its relatively slow convergence has inspired a search for other point sets whose discrepancy is lower than that expected for truly random points. Such low-discrepancy point sets and low-discrepancy sequences have developed into a veritable industry, and sequences with, asymptotically, very low discrepancy are now available, especially for problems with very many variables [8]. For point sets that are extracted as the first $N$ elements of such a sequence, though, one is usually still compelled to compute the discrepancy numerically, and compare it to the expectation for random points in order to show that the point set is indeed 'better than random'. This implies, however, that one has to know, for a given discrepancy, its expectation value for truly random points, or preferably even its probability density. In Refs. [9-12] we have solved this problem for large classes of discrepancies. Although computable, the resulting distributions are typically not very illuminating. The exception is usually the case where the number of dimensions of the integration problem becomes very large, in which case a normal distribution often arises $[5,13]$. In this paper, we investigate this phenomenon in more detail, and we shall describe the conditions under which this 'law of large dimensions' applies.

The layout of this paper is as follows. In Section 2, we define the general structure of a discrepancy related to a class of integrands of which it is an average-case complexity. We show how to derive the probability density of this discrepancy when viewed as a stochastic variable defined on sets of truly random points. Then, we investigate the conditions under which this density approaches a normal density. Finally, in Section 3 we apply our results to a few toy-models and standard choices of discrepancy. A number of technical points are collected in the various appendices. Throughout this discussion, we shall only consider the asymptotic limit of a very large number of integration points. This implies that, in this paper, 'Ne cannot make any statements on how the number of points has to approach infinity with respect to the number of dimensions, as was for instance done in Ref. [13]. In Ref. [15], we repair this defect, and shall be able to show which precise combination of limits has to be taken.

\section{General definitions and statements}

To set the stage, we shall always consider the integration region to be the $s$-dimensional unit hypercube $K=$ $[0,1)^{s}$. The point set $X_{N}$ consists of $N$ points $x_{k}^{\mu}$, where $k=1,2, \ldots, N$ labels the points and $\mu=1,2, \ldots, s$ their coordinates.

\subsection{Quadratic discrepancy and complexity}

We will define quadratic discrepancies as the average-case complexity of an integration problem in terms of its averaged squared integration error [6]. For the given class of real-valued functions $f(x)$, with $x \in K$, let a measure $d \mu(f)$ on the class of functions be given, such that the one- and two-point connected Green's functions are given by

$$
\begin{aligned}
& \int f(x) d \mu(f)=0, \\
& \int f\left(x_{1}\right) f\left(x_{2}\right) d \mu(f)=\int_{L} h\left(y ; x_{1}\right) h\left(y ; x_{2}\right) d \mu(y) .
\end{aligned}
$$

Here we assume that we can define a function $h(y, x)$ and measure $d \mu(y)$ over some space $L$ such that the above expression makes sense. The variable $y$ has to be suitably defined; it may be a continuous variable with a continuous integration measure $d \mu(y)$, or a discrete variable, in which case $\int d \mu(y)$ reduces to a sum over an enumerable set of discrete values, such as a lattice: all cases we will consider in this article can be expressed 
in terms of an enumerable set of discrete values. For the moment we will stick to the more general notation of $d \mu(y)$. We define the quadratic discrepancy ${ }^{4} D_{N}$ as follows [4]:

$$
\begin{aligned}
& \frac{D_{N}}{N}=\int \eta^{2}[f] d \mu(f), \\
& \eta[f]=\frac{1}{N} \sum_{k=1}^{N} f\left(x_{k}\right)-\int_{K} f(x) d x .
\end{aligned}
$$

In Ref. [4] it was shown that, if the function measure $d \mu(f)$ is Gaussian in the sense that the only nonvanishing connected Green's function is the two-point function, then the integration error will be normally distributed with zero mean and variance equal to $D_{N} / N$. The discrepancy $D_{N}$ can be written as

$$
\begin{aligned}
& D_{N}=\frac{1}{N} \sum_{k . l=1}^{N} \beta\left(x_{k}, x_{l}\right), \\
& \beta\left(x_{k}, x_{l}\right)=\int_{L} \omega\left(y ; x_{k}\right) \omega\left(y ; x_{l}\right) d \mu(y), \quad \omega\left(y ; x_{k}\right)=h\left(y ; x_{k}\right)-\int_{K} h(y ; x) d x .
\end{aligned}
$$

In fact, $D_{N}$ measures how well the function $h(y ; \cdot)$ is integrated by the point set $X_{N}$, averaged over $y$. Notice that $D_{N}$ is nonnegative by construction, and that for an infinite equidistribeted sequence, $\lim _{N \rightarrow \infty} D_{N}=0$. Moreover, the expected value of $D_{N}$ for a set of $N$ truly randor: points in $K$ is given by

$$
\mathrm{E}\left[D_{N}\right]=\int \mathrm{V}[f] d \mu(f)=\iint_{L} \omega(y ; x)^{2} d x d \mu(y),
$$

where $E[\cdot]$ denotes the expectation value w.r.t. the uniform distribution over the ensemble of truly random point sets with $N$ points. We shall always assume this expectation value to be a finite quantity, otherwise this discrepancy cannot meaningfully be used for truly random points.

In our approach to calculate discrepancy distributions, we will also use the higher momenta $\mathrm{E}\left[D_{N}^{m}\right]$ ( $m=$ $1,2,3, \ldots)$, which therefore have to be assumed to be finite ${ }^{5}$. We will also define some useful functions $\beta_{k}$ and $\Gamma_{k}$,

$$
\begin{aligned}
& \beta_{k}\left(x_{1}, x_{2}\right)=\int_{K} \beta\left(x_{1}, x\right) \beta_{k-1}\left(x, x_{2}\right) d x, \\
& \Gamma\left(y_{1}, y_{2}\right)=\int_{K} \omega\left(y_{1} ; x\right) \omega\left(y_{2} ; x\right) d x, \\
& \Gamma_{k}\left(y_{1}, y_{2}\right)=\int_{L} \Gamma\left(y_{1}, y\right) \Gamma_{k-1}\left(y, y_{2}\right) d \mu(y),
\end{aligned}
$$

with $\beta_{1}=\beta$ and $\Gamma_{1}=\Gamma$. The function $\Gamma$ is in a certain sense dual to the function $\beta$. It will be more convenient to use, because the variable $y$ is often an element of a countable set and $\Gamma$ can be seen as a matrix, with $\Gamma_{k}\left(y_{1}, y_{2}\right)=\Gamma^{k}\left(y_{1}, y_{2}\right)$.

\footnotetext{
${ }^{4}$ Note that we extract a factor $N$ out of the definition of the discrepancy as compared to other definitions in the literature such that the discrepancy averaged over the ensemble of truly random point sets is independent of $N$.

${ }^{5}$ For the discrepancies we discuss, this is a valid assumption.
} 


\subsection{Gaussian measures on a countable basis}

In this article we will consider function classes with functions $f$ that can be written as linear combinations of a countable set of basis functions $\left\{u_{n}\right\}$,

$$
f(x)=\sum_{n} v_{n} u_{n}(x)
$$

Often we will refer to the basis functions as modes. We assume that integrals over combinations $u_{n_{1}}(x) u_{n_{2}}(x) \ldots$ exist and introduce the parameters

$$
w_{n}=\int_{K} u_{n}(x) d x \text { and } a_{m, n}=\int_{K} u_{m}(x) u_{n}(x) d x .
$$

The variance of $f$ can then be written as

$$
\mathrm{V}[f]=\int_{K} f(x)^{2} d x-\left(\int_{K} f(x) d x\right)^{2}=\sum_{m, n} v_{m} v_{n}\left(a_{m, n}-w_{m} w_{n}\right) .
$$

A Gaussian measure on the class of functions is obtained by taking

$$
d \mu(f)=\prod_{n} \frac{\exp \left(-v_{n}^{2} / 2 \sigma_{n}^{2}\right)}{\sqrt{2 \pi \sigma_{n}^{2}}} d v_{n} .
$$

For the measure to be suitably defined, the strengths $\sigma_{n}$ have to satisfy certain restrictions. In particular, we want the functions $f$ to be quadratically integrable on the average. The reasonable requirement of $E\left[D_{N}\right]$ to exist ensures that the variance of the functions $f$ exists on the average and thus imposes a condition on the strengths

$$
\mathrm{E}\left[D_{N}\right]=\int \mathrm{V}[f] d \mu(f)=\sum_{n} \sigma_{n}^{2} \mathrm{~V}\left[u_{n}\right]
$$

Now we can use the formalism of the previous section to construct the discrepancy. The two-point connected Green's function is given by

$$
\int f\left(x_{1}\right) f\left(x_{2}\right) d \mu(f)=\sum_{n} \sigma_{n}^{2} u_{n}\left(x_{1}\right) u_{n}\left(x_{2}\right),
$$

which is nothing but a spectral representation. The functions $h$ and $\omega$ can be taken equal to

$$
h_{n}(x)=\sigma_{n} u_{n}(x), \quad \omega_{n}(x)=\sigma_{n}\left(u_{n}(x)-w_{n}\right),
$$

where the variable $y$ is replaced by the countable index $n$. The function $\beta$ and the matrix $\Gamma$ are given by

$$
\begin{aligned}
& \beta\left(x_{1}, x_{2}\right)=\sum_{n} \sigma_{n}^{2}\left(u_{n}\left(x_{1}\right)-w_{n}\right)\left(u_{n}\left(x_{2}\right)-w_{n}\right), \\
& \Gamma_{m, n}=\sigma_{m} \sigma_{n}\left(a_{m, n}-w_{m} w_{n}\right) .
\end{aligned}
$$

Note that we have for the trace of $\Gamma_{m, n}$,

$$
\operatorname{Tr}(\Gamma)=\sum_{n} \sigma_{n}^{2} \mathrm{~V}\left[u_{n}\right]=\mathrm{E}\left[D_{N}\right]
$$




\subsection{General form of discrepancy distributions}

We now turn to the problem of computing the probability density of such a discrepancy when the $N$ points are (independently and uniformly) randomly distributed over $K$. Introducing the Dirac $\delta$-distribution and its representation as a Laplace transform, we may write the probability density $H(t)$ for the value $t$ of discrepancy $D_{N}=D_{N}\left(x_{1}, x_{2}, \ldots, x_{N}\right)$ as

$$
\begin{aligned}
H(t) & =\int_{K} \int_{K} \cdots \int_{K} \delta\left(D_{N}\left(x_{1}, x_{2}, \ldots, x_{N}\right)-t\right) d x_{1} d x_{2} \cdots d x_{N} \\
& =\frac{1}{2 \pi i} \int_{-i \infty}^{+i \infty} e^{-z t} G_{0}(z) d z
\end{aligned}
$$

where the $z$ integration runs along the imaginary axis, and $G_{0}(z)$ is the moment-generating function

$$
G_{0}(z)=\mathrm{E}\left[e^{z D_{N}}\right]=\sum_{m \geq 0} \frac{z^{m}}{m !} \mathrm{E}\left[D_{N}^{m}\right] .
$$

At this point it may be useful to note that, since $D_{N}$ is nonnegative by construction, we must have $H(t)=0$ for $t<0$, and hence no singular point of $G_{0}(z)$ may have a negative real part.

The task is, now, to compute $G_{0}(z)$ as a series expansion around $z=0$. In Refs. $[5,9,10,12]$ we have shown how Feynman diagrams may be usefully employed to do this in a systematic way in the limit of large $N$. In this paper we shall restrict ourselves to the leading behaviour $N \rightarrow \infty$, in which limit we have

$$
\log \left(G_{0}(z)\right)=\sum_{k>0} \frac{(2 z)^{k}}{2 k} R_{k}, \quad R_{k}=\int_{K} \beta_{k}(x, x) d x=\int_{L} \Gamma_{k}(y, y) d \mu(y) .
$$

In those cases where the $y$ variables are discrete and enumerable, $\Gamma$ can be written as a real symmetric matrix, and then we simply have

$$
G_{0}(z)=(\operatorname{det}(1-2 z \Gamma))^{-1 / 2}, \quad R_{k}=\operatorname{Tr}\left(\Gamma^{k}\right) .
$$

We shall - symbolically - employ the matrix and trace notation for the continuous case as well. In general, we have

$$
\begin{aligned}
& \Gamma_{1}\left(y_{1}, y_{2}\right)=A\left(y_{1}, y_{2}\right)-B\left(y_{1}\right) B\left(y_{2}\right), \\
& A\left(y_{1}, y_{2}\right)=\int_{K} h\left(y_{1} ; x\right) h\left(y_{2} ; x\right) d x, \quad B(y)=\int_{K} h(y ; x) d x .
\end{aligned}
$$

In many cases (cf. the case of orthonormal functions bases), we have $B(y)=0$, but this is not necessary. In general, then, $\operatorname{Tr}\left(\Gamma^{k}\right)$ consists of $2^{k}$ terms. However, as we show in Appendix A, we can combine them nicely, so that we find

$$
\begin{aligned}
& G_{0}(z)=\exp (\psi(z)) / \sqrt{\chi(z)} \\
& \psi(z)=\sum_{k>0} \frac{(2 z)^{k}}{2 k} \operatorname{Tr}\left(A^{k}\right) \\
& \chi(z)=1+\sum_{k>0}(2 z)^{k} \operatorname{Tr}\left(B A^{k-1} B\right) .
\end{aligned}
$$




\subsection{Standardized variables and the Gaussian limit}

We now have derived the expression for $G_{0}(z)$ in the large- $N$ limit. Given the form of $\Gamma\left(y_{1}, y_{2}\right)$, we can now compute $H(t)$ for given discrepancy $t$, if only numerically; in fact this was done for the $L_{2}$ stardiscrepancy in Ref. [5] for several dimensionalities. In some special cases, $H(t)$ can even be given as an analytic expression $[10,11]$. Here, however, we are interested in possible Gaussian limits, and therefore it is useful to replace the value $t$ of the discrepancy by the standardized variable $\xi$ as follows:

$$
t=t(\xi)=\mathrm{E}\left[D_{N}\right]+\xi \sqrt{\mathrm{V}\left[D_{N}\right]},
$$

where the expectation $\mathrm{E}\left[D_{N}\right]$ and variance $\mathrm{V}\left[D_{N}\right]$ of the discrepancy (which equal $R_{1}$ and $2 R_{2}$, respectively) are taken out such that the stochastic variable $\xi$ always has expectation zero and variance 1 . By furthermore going over from $z$ to $u=z / \sqrt{2 R_{2}}$ in Fq. (19), we can write the probability density $\widehat{H}(\xi)$ of $\xi$ as

$$
\begin{aligned}
& \hat{H}(\xi)=H(t(\xi)) \frac{d t(\xi)}{d \xi} \\
&=\frac{\exp \left(-\xi^{2} / 2\right)}{2 \pi i} \int_{-i \infty}^{+i \infty} d u \exp \left(\frac{1}{2}(u-\xi)^{2}+\sum_{k \geq 3} u^{k} \frac{2^{(k-2) / 2}}{k} \gamma_{k}^{1 / 2}\right), \\
& \gamma_{k}=R_{k}^{2} / R_{2}^{k} .
\end{aligned}
$$

All information on the particulars of the discrepancy are now contained in the constants $\gamma_{k}$ and we have that the probability density of $\xi$ approaches the normal density whenever $\gamma_{k} \rightarrow 0$ for all $k \geq 3$. It remains to examine under what circumstances this can happen.

\subsection{A law of large number of modes}

Let us assume, for the moment, that the matrix $\Gamma$ is indeed a real symmetric matrix. This is for instance the case for Gaussian measures on a countable basis. Moreover, since we know that $G_{0}(z)$ has no singularities for negative values of $\operatorname{Re} z$, the eigenvalues of $\Gamma$ are also nonnegative, and we may write

$$
\operatorname{Tr}\left(\Gamma^{k}\right)=\sum_{n} \lambda_{n}^{k}, \quad \gamma_{k}=\left(\sum_{n} \lambda_{n}^{k}\right)^{2}\left(\sum_{n} \lambda_{n}^{2}\right)^{-k}, \quad \lambda_{n} \geq 0 .
$$

where the various eigenvalues have been denoted by $\lambda_{n}$. Note that the sum may run over a finite or an infinite number of eigenvalues, but all these sums inust converge since $\mathrm{E}\left[D_{N}\right]$ is finite. Note, moreover, that $\gamma_{k}$ is homogeneous of degree zero in the $\lambda_{n}$ : therefore, any scaling of the eigenvalues by a constant does not influence the possible Gaussian limit (although it will, of course, affect the mean and variance of $D_{N}$ ).

We now proceed by noting that $\gamma_{k+1} \leq \gamma_{k}$, because

$$
\left(\sum_{n} \lambda_{n}^{k+1}\right)^{2} \leq\left(\sum_{n} \lambda_{n}^{2 k}\right)\left(\sum_{n} \lambda_{n}^{2}\right) \leq\left(\sum_{n} \lambda_{n}^{k}\right)^{2}\left(\sum_{n} \lambda_{n}^{2}\right),
$$

where the first inequality is simply the Schwarz inequality, and the second one holds because the $\lambda_{n}$ are nonnegative. This means that $\gamma_{k}$ will approach zero for $k>3$, whenever $\gamma_{3}$ approaches zero. To see when this happens, we define

$$
x_{n}=\frac{\lambda_{n}}{\sqrt{\sum_{m} \lambda_{m}^{2}}}, \quad x=\max _{n} x_{n}
$$


so that $\sum_{n} x_{n}^{2}=1$. It is then trivial to see that

$$
x^{3} \leq \gamma_{3}^{1 / 2} \leq x
$$

from which we derive that the necessary and sufficient condition for the discrepancy distribution to approach a Gaussian is that

$$
C=\frac{\lambda^{2}}{\sum_{n} \lambda_{n}^{2}} \rightarrow 0, \quad \lambda=\max _{n} \lambda_{n} .
$$

The Gaussian limit is thus seen to be equivalent to the statement that even the largest eigenvalue becomes unimportant. Clearly, a necessary condition for this is that the total number of nonvanishing eigenvalues (number of modes) approaches infinity. Incidentally, the condition (31) also implies that

$$
\lambda \rightarrow 0, \quad \sum_{n} \lambda_{n}^{2} \rightarrow 0,
$$

for all those discrepancies that have $\mathrm{E}\left[D_{N}\right]=\sum_{n} \lambda_{n}=1$. This is eminently reasonable, since a distribution centered around 1 and (by construction) vanishing for negative argument can only approach a normal distribution if its variance approaches zero. On the other hand, the condition $\lambda \rightarrow 0$ is by itself not sufficient, as proven by a counterexample given in Appendix B.

Another piece of insight can be obtained if we allow the eigenvalues to take on random values. We may introduce the rather dizzying concept of an ensemble of different definitions of discrepancy, each characterized by its set of eigenvalues (all nonnegative) $\lambda=\left\{\lambda_{1}, \lambda_{2}, \ldots, \lambda_{M}\right\}$, with the usual constraint that they add up to 1; we keep $M$ finite for simplicity. A natural probability measure on this ensemble is given by the probability density $P_{\lambda}(\lambda)$ of the random vector $\lambda$,

$$
P_{\lambda}(\lambda)=\Gamma(M) \delta\left(\sum_{n=1}^{M} \lambda_{n}-1\right)
$$

Here $\Gamma$ denotes Eulers gamma-function. It is easily computed that the expectation and variance of $R_{k}=\sum_{n} \lambda_{n}^{k}$ - given, for large $M$, by

$$
\mathrm{E}\left[R_{k}\right] \sim \frac{k !}{M^{k-1}}, \quad \mathrm{~V}\left[R_{k}\right] \sim \frac{(2 k) !-\left(1+k^{2}\right)(k !)^{2}}{M^{2 k-1}},
$$

so that the $R_{k}$ become sharply peaked around their expectation for large $M$. In that case, we have

$$
\gamma_{3} \sim \frac{9}{2 M},
$$

and we see that, in the above sense, almost all discrepancies have a Gaussian distribution in the limit where $M$, the number of modes, approaches infinity.

\section{Applications to different examples}

\subsection{Fastest approach to a Gaussian limit}

We now examine the various definitions of discrepancies, and assert their approach to a Gaussian limit. Usually this is envisaged, for instance in Ref. [13], as the limit where the dimensionality $s$ of $K$ becomes 
very large. But, as we have shown, this is only a special case of the more general situation where the number of relevant modes becomes very large: another possible case is that where, in one dimension, the number of modes with essentially equal strength $\sigma_{n}$ becomes very large. As an illustration, consider the case where the basis functions with the Gaussian measure are orthonormal and $M$ of the nontrivial modes have equal strength $\sigma_{n}^{2}=1 / M$, and the rest have strength zero. The moment-generating function then takes on a particularly simple form, and so does the discrepancy distribution [11],

$$
\log \left(G_{0}(z)\right)=-\frac{M}{2} \log \left(1-\frac{2 z}{M}\right), \quad H(t)=\frac{(M / 2)^{M / 2}}{\Gamma(M / 2)} t^{M / 2-1} e^{-M / 2} .
$$

It is easily seen that the gamma-distribution $H(t)$ approaches a normal one when $M$ becomes very large. At the same time, we see the 'physical' reason behind this: it is the fact that the singularity of $G_{0}(z)$ in the complex plane (in the more general case, the singularity nearest to $z=0$ ) moves away to infinity. One observation is relevant here: in Eq. (26), we have kept the integration over $u$ along the imaginary axis $\operatorname{Re} u=0$. We might consider performing a saddle-point integration, with a nonvanishing value of $\operatorname{Re} u$. That may give us, for a finite number of modes, a good approximation to the actual form of $H(t)$. It is quite possible, and, indeed, it happens in the above equal-strength model, that this approximation is already quite similar to a Gaussian. In the equal-strength model, a saddle-point approximation for $H(t)$ gives precisely the form of Eq. (36), the only difference being that $\Gamma(M / 2)$ is replaced by its Stirling approximation. On the other hand, for not-so-large $M$, this form is not too well approximated by a Gaussian centered around $t=1$, since the true maximum resides at $t=1-2 / M$. Nevertheless, in this paper we are only interested in the limiting behaviour of $H(t)$, and we shall stick to the use of condition (31) as an indicator of the Gaussian limit.

One interesting remaining observation is the following. For any finite number $M$ of eigenvalues $\lambda_{n}$ ( $n=$ $1,2, \ldots, M)$, the smallest value of the indicator $C=\lambda^{2} / \sum_{n} \lambda_{n}^{2}$ is obtained when $\lambda_{n}=1 / M$ for all $n$. In this sense, the equal-strengths model gives, for finite $M$, that discrepancy distribution that is closest to a Gaussian.

\section{2. $L_{2}$ star-discrepancy and the Wiener measure}

Here we shall discuss the standard $L_{2}$ star-disc:epancy [2]. We start with a formulation of the problem using a continuous variable $y$ on $K$, and $d \mu(y)=d y$. The function $h$ is given by

$$
h\left(y ; x_{k}\right)=\prod_{\mu=1}^{s} \theta\left(x_{k}^{\mu}<y^{\mu}\right),
$$

where we have introduced the $\theta(\cdot)$ as the logical step-function ${ }^{6}$. The Gaussian function measure corresponding to this discrepancy is therefore seen to be defined by

$$
\int f\left(x_{1}\right) f\left(x_{2}\right) d \mu(f)=\prod_{\mu=1}^{s} \min \left(1-x_{1}^{\mu}, 1-x_{2}^{\mu}\right),
$$

which we can recognize as that variation of the standard Wiener sheet measure in which the function $f(x)$ is pinned down at $x=(1,1, \ldots, 1)$ rather than at $x=(0,0, \ldots, 0)$. This is the content of the original Woźniakowski lemma from Ref. [6].

A formulation of this discrepancy in terms of a Gaussian measure on a countable basis can be constructed by realizing that a spectral representation of the integration kernel $g\left(x_{1}, x_{2}\right)=\prod_{\mu=1}^{s} \min \left(x_{1}^{\mu}, x_{2}^{\mu}\right)$ exists [17] and is given by

\footnotetext{
${ }^{6}$ The logical step-function $\theta(P)$ of an expression $P$ is equal to 1 if the $P$ is true, and 0 if $P$ is false. Therefore $\theta(x<y)$ is in fact equal to the Heavyside function $\theta(y-x)$.
} 


$$
g\left(x_{1}, x_{2}\right)=\sum_{n \geq 0} \sigma_{n}^{2} u_{n}\left(x_{1}\right) u_{n}\left(x_{2}\right)
$$

where the functions $u_{n}$ are given by

$$
u_{n}(x)=2^{s / 2} \prod_{\mu=1}^{s} \sin \left(r\left(n_{\mu}\right) \frac{\pi}{2} x^{\mu}\right),
$$

and the strengths $\sigma_{n}^{2}$ by

$$
\sigma_{n}^{2}=\left(\frac{4}{\pi^{2}}\right)^{s} \prod_{\mu=1}^{s} \frac{1}{r\left(n_{\mu}\right)^{2}}, \quad r(n)=(2 n+1) \theta(n \geq 0) .
$$

Because a Gaussian measure is completely defined by its two-point Green's function, the measure defined by the basis functions $u_{n}$ is equivalent with the Wiener measure. In Appendix C we show that the discrepancy defined using this formulation of the Gaussian measure on a countable basis is equivalent to the $L_{2}$ star-discrepancy.

The functions $u_{n}$ are orthonormal, and we have

$$
w_{n i}=2^{s / 2} \sigma_{n} \text { and } a_{m, n}=\delta_{m, n} .
$$

The matrix $\Gamma$ is given by

$$
\Gamma_{m, n}=\sigma_{m}^{2} \delta_{m, n}-2^{s} \sigma_{m}^{2} \sigma_{\pi}^{2},
$$

and an eigenvalue equation for the eigenvalues $\lambda$ can be written down easily,

$$
\prod_{n}\left(\sigma_{n}^{2}-\lambda\right)\left[1-2^{s} \sum_{m} \frac{\sigma_{m}^{4}}{\sigma_{m}^{2}-\lambda}\right]=0
$$

In value the strengths $\sigma_{n}$ are degenerate. Labelling the strengths with different values by $\sigma_{p}$ with $p=$ $\prod_{\mu=1}^{s} r\left(n_{\mu}\right)$, the degeneracy is given by

$$
Q_{w}(p)=\sum_{n \geq 0} \theta\left(p=\prod_{\mu=1}^{s} r\left(n_{\mu}\right)\right) .
$$

So $\lambda=\sigma_{p}^{2}$ is a solution to the eigenvalue equation with a $\left(Q_{w}(p)-1\right)$-fold degenerary. If we factorize these solutions we obtain the following equation for the remaining eigenvalues:

$$
1-2^{s} \sum_{p} Q_{\mathrm{w}}(p) \frac{\sigma_{p}^{4}}{\sigma_{p}^{2}-\lambda}=0
$$

Some assertions concerning the remaining eigenvalues can be made using this equation. On inspection, it can be seen that there are no negative solutions, nor solutions larger than $\sigma_{1}^{2}$, so that $\sigma_{1}^{2}$ can be used as an upper bound of the eigenvalues of $\Gamma$. If we order the $\lambda$ such that $\lambda_{1} \geq \lambda_{3} \geq \cdots$, then $\sigma_{1}^{2} \geq \lambda_{1} \geq \sigma_{3}^{2} \geq \lambda_{3} \geq \cdots$. This implies that $\operatorname{Tr}\left(\Gamma^{k}\right)=\sum_{p} Q_{w}(p) \sigma_{p}^{2 k}-\epsilon$ where $0 \leq \epsilon \leq \sigma_{1}^{2 k}$. Note that $\sum_{p} Q_{w}(p) \sigma_{p}^{2 k}=\operatorname{Tr}\left(g^{k}\right)$ so that traces of $g^{k}$ are upper bounds of traces of $\Gamma^{k}$. Now we have

$$
\operatorname{Tr}\left(g^{k}\right)=\left(\frac{4}{\pi^{2}}\right)^{k s} \xi(2 k)^{s}, \quad \xi(p)=\sum_{n \geq 0} \frac{1}{(2 n+1)^{p}},
$$


and therefore for $k \geq 3$,

$$
\gamma_{k} \leq\left(\frac{\xi(2 k)^{2}}{\xi(4)^{k}}\right)^{s}\left(1-2\left(\frac{4}{5}\right)^{s}+\left(\frac{2}{3}\right)^{s}\right)^{-k}
$$

The second factor decreases monotonically from $(15)^{k}$ for $s=1$ to one as $s \rightarrow \infty$; for the first factor, we note that $1<\xi(2 k)<\xi(4)$ for all $k>2$. Therefore, $\gamma_{k}$ can be made arbitrarily small by choosing $s$ large enough, and the Gaussian limit of high dimensionality is proven. Note, however, that the approach is not particularly fast: for large $s$, we have $\gamma_{3} \sim(24 / 25)^{s} \sim \exp (-s / 25)$, so that $s$ has to become of the order of one hundred or so to make the Gaussian behaviour manifest. In fact, this was already noted by explicit numerical computation in Ref. [5].

\subsection{Diaphony}

\subsubsection{General definition}

In one dimension the discrepancy defined through a Gaussian measure on a countable basis is called diaphony if the basis functions $\left\{u_{n}\right\}$ are such that

$$
w_{n}=0 \text { and } a_{m, n}=\delta_{m, n} \text {. }
$$

These relations are typically satisfied when the functions are orthonormal and $u_{0}(x)=1$ is one of the basis functions. The matrix $\Gamma$ is given by

$$
\Gamma_{m, n}=\sigma_{n}^{2} \delta_{m, n},
$$

so the eigenvalues are given by the squares $\sigma_{n}^{2}$ of the strengths itself. An extension to more dimensions can be obtained by taking products $u_{n}(x)=\prod_{\mu=1}^{s} u_{n_{\mu}}\left(x^{\mu}\right)$ of one-dimensional functions. However, in contrast to the Wiener sheet measure that underlies the $L_{2}$ star-discrepancy, there appears to be no 'natural' generalization of the strengths $\sigma_{n}$ to more dimensions, and therefore we shall discuss various possibilities. In general, we want to let the strength $\sigma_{n}$ depend on a global property of the vector $n$, for instance, the product of the components, or the sum of the components: we shall call such alternatives clusterings.

\subsubsection{Fourier diaphony}

As an application of the above, let us consider the orthonormal functions defined by the one-dimensional factors

$$
u_{2 k-1}(x)=\sqrt{2} \sin (2 \pi k x), \quad u_{2 k}(x)=\sqrt{2} \cos (2 \pi k x), \quad k=1,2,3, \ldots
$$

Furthermore, it is useful to take the $\sigma_{n}$ such that the sine and cosine modes with equal wavenumber appear with equal coefficients. Let us define

$$
k(n)=k \theta(2 k-1 \leq n \leq 2 k) .
$$

We require that $\sigma_{n}$ only depends on $n$ via $\boldsymbol{k}(\boldsymbol{n})$,

$$
\sigma_{n}=\sigma(k(n)), \quad \boldsymbol{k}(\boldsymbol{n})=\left(k\left(n_{1}\right), k\left(n_{2}\right), \ldots, k\left(n_{s}\right)\right) .
$$

In that case, the diaphony is equal to

$$
D_{N}=\frac{1}{N} \sum_{k \neq 0} \sigma^{2}\left(\left|k_{1}\right|,\left|k_{2}\right|, \ldots,\left|k_{s}\right|\right)\left|\sum_{l=1}^{N} e^{2 i \pi k \cdot x_{l}}\right|^{2},
$$


where, this time, the vector $\boldsymbol{k}$ runs over the whole integer lattice except the origin; and it has the appealing property that the value of the Fourier discrepancy is the same for point sets differing only by a translation mod 1 ; the $L_{2}$ star-discrepancy does not have this nice property.

\subsubsection{Fourier diaphony with product clustering}

One of the most straightforward generalizations of the Fourier diaphony, and the choice made in Ref. [3], is to let $\sigma_{n}$ depend on the product of the frequency components,

$$
\sigma_{n}^{2}=\frac{1}{\left(1+\pi^{2} / 3\right)^{s}-1} \prod_{\mu=1}^{s} \frac{1}{r\left(n_{\mu}\right)^{2}}, \quad r(n)=\theta(n=0)+k(n) \theta(n>0) .
$$

The normalization of the $\sigma_{n}$ ensures that $\mathrm{E}\left[D_{N}\right]=1$, independent of $s$. In this case, keeping in mind that sines and cosines occur with equal strength, we have to consider the multiplicity function

$$
Q_{F}^{\mathrm{II}}(p)=\sum_{n \geq 0} \theta\left(p=\prod_{\mu} r\left(n_{\mu}\right)\right)
$$

Actually, before assigning a strength $\sigma_{n}$, or rather $\sigma_{p}^{2}$, we have to know the behaviour of $Q_{F}^{\mathrm{n}}(p)$ in order to ensure convergence of $\mathrm{E}\left[D_{N}\right]$. In order to do so, we introduce the Dirichlet generating function for $Q_{F}^{\Pi}(p)$,

$$
F_{s}^{(1)}(x)=\sum_{p>0} \frac{Q_{F}^{\Pi}(p)}{p^{x}}=(1+2 \zeta(x))^{s},
$$

where we use the Riemann $\zeta$ function. Since this function (and, therefore, $F_{s}^{(1)}(x)$ as well) converges for all $x>1$, we are ensured that $Q_{F}^{\Pi}(p)$ exceeds the value $c p^{1+\epsilon}$ at most for a finite number of values of $p$, for all positive $c$ and $\epsilon$. This is proven in Appendix D. It is therefore sufficient that $\sigma_{p}^{2}$ decreasrs as a power (larger than 1) of $p$. In fact, taking

$$
\sigma_{p}^{2}=c p^{-\beta}, \quad \beta>1,
$$

we immediately have that

$$
R_{k}=\sum_{n>0} \sigma_{n}^{2 k}=\sum_{p>0} Q_{F}^{\Pi}(p) \sigma_{p}^{2 k}-\sigma_{1}^{2 k}=c^{k}\left[(1+2 \zeta(k \beta))^{s}-1\right],
$$

which, for given $\beta$, fixes $c$ such that $R_{1}=\mathrm{E}\left[D_{N}\right]=1$, and, moreover, gives

$$
\gamma_{3} \sim a(\beta)^{s} \quad \text { as } s \rightarrow \infty, \quad a(\beta)=\frac{(1+2 \zeta(3 \beta))^{2}}{(1+2 \zeta(2 \beta))^{-3}}
$$

As indicated above, in Ref. [3] the value $\beta=2$ is used, with $a(2) \sim 0.291$. The supremum of $a(\beta)$ equals $1 / 3$, as $\beta \rightarrow \infty$, and the (more interesting) infimum is $a(1)$, about 0.147 . We conclude that, for all diaphonies of the above type, the Gaussian limit appears for high dimensionality. For large $\beta$, where the higher modes are greatly suppressed, the convergence is slowest, in accordance with the observation that the 'equal-strength' model gives the fastest convergence; however, the convergence is still much faster than for the $L_{2}$ star-discrepancy, and the Gaussian approximation is already quite good for $s \sim 4$. The fastest approach to the Gaussian limit occurs when we force all modes to have as equal a strength as is possible within the constraints on the $\beta$. The difference between the supremum and infimum of $a(\beta)$ is, however, not much more than a factor of 2 . 
Another possibility would be to let $\sigma_{p}^{2}$ depend exponentially on $p$. In that way one can ensure convergence of the $R_{k}$ while at the same time enhancing as many low-frequency modes as possible. It is proven in Appendix $D$ that the function

$$
F_{s}^{(2)}(x)=\sum_{p>0} Q_{F}^{\Pi}(p) x^{p}
$$

has radius of convergence equal to one, and therefore we may take $\sigma_{p}^{2}=\left(\beta^{\prime}\right)^{p}$ with $\beta^{\prime}$ between zero and one. If we choose $\beta^{\prime}$ to be very small, we essentially keep only the modes with $p=1$, and therefore in that case we have $\gamma_{3} \sim 1 /\left(3^{s}-1\right)$. This is of course in reality the same type of discrepancy as the above one, with $\beta \rightarrow \infty$. On the other hand, taking $\beta^{\prime} \rightarrow 1$ we arrive at $\gamma_{3} \rightarrow 0$ (see, again, Appendix D). The difference with the first model is, then, that we can approach the Gaussian limit arbitrarily fast, at the price, of course, of having a function $\beta\left(x_{k}, x_{l}\right)$ that is indistinguishable from a Dirac $\delta$-distribution in $x_{k}-x_{l}$, and hence meaningless for practical purposes.

\subsubsection{Fourier diaphony with sum clustering}

In the above, we have let the strength $\sigma_{n}$ depend on the product of the various $r\left(n_{\mu}\right)$. This can be seen as mainly a matter of expediency, since the generalization to $s>1$ is quite simple in that case. From a more 'physical' point of view, however, this grouping of the $\sigma$ is not so attractive, if we keep in mind that each $n$ corresponds to a mode with wave vector $k(n)$. Under the product rule, wave vectors differing only in their direction but with equal length may acquire vastly different weights: for instance, $k=(m \sqrt{s}, 0,0, \ldots)$ and $k=(m, m, m, \ldots)$ have equal Euclidean length, $m \sqrt{s}$, but their strengths under the product rule are $1 / \mathrm{sm}^{2}$ and $1 / m^{2 s}$, respectively. This lack of 'rotational' symmetry could be viewed as a drawback in a discrepancy distinguished by its nice 'translational' symmetry. One may attempt to soften this problem by grouping the strengths $\sigma_{n}$ in another way, for instance by taking

$$
\sigma_{n}=\sigma\left(\sum_{\mu} k\left(n_{\mu}\right)\right)
$$

so that $\sigma$ depends on the sum of the components rather than on their product. The multiplicity of a given strength now becomes, in fact, somewhat simpler,

$$
Q_{r}^{\Sigma}(p)=\sum_{n>0} \theta\left(p=\sum_{\mu=1}^{s} k\left(n_{\mu}\right)\right)=\sum_{m \geq 0}\left(\begin{array}{c}
s \\
m
\end{array}\right)\left(\begin{array}{c}
s-1+p-m \\
p-m
\end{array}\right),
$$

where the last identity follows from the generating function

$$
F_{s}^{(3)}(x)=\sum_{p \geq 0} Q_{F}^{\Sigma}(p) x^{p}=\left(\frac{1+x}{1-x}\right)^{s}
$$

This also immediately suggests the most natural form for the strength: $\sigma_{n}^{2}=\beta^{p}$, where $p$ is $\sum_{\mu} k\left(n_{\mu}\right)$ as above. We see that $R_{1}$ converges as long as $\beta<1$, and moreover,

$$
\gamma_{3}=\frac{\left[\left(\frac{1+\beta^{3}}{1-\beta^{3}}\right)^{s}-1\right]^{2}}{\left[\left(\frac{1+\beta^{2}}{1-\beta^{2}}\right)^{s}-1\right]^{3}} \sim a(\beta)^{s},
$$

where $a(\beta)$ has supremum $a(0)=1$, and decreases monotonically with increasing $\beta$. For $\beta$ close to one, we have $a(\beta) \sim 4(1-\beta) / 9$, so that the Gaussian limit can be reached as quickly as desired (again with the reservations mentioned above). At the other extreme, note that for very small $\beta$ we shall have 


$$
\gamma_{3} \sim \frac{1}{2 s} \text { if } s \beta^{2} \ll 1
$$

This just reflects the fact that, for extremely small $\beta$, only the $2 s$ lowest nontrivial modes contribute to the discrepancy; and even in that case the Gaussian limit is attained, although much more slowly. The criterion that determines whether the behaviour of $\gamma_{3}$ with $s$ and $\beta$ is exponential or of type $1 / 2 s$ is seen to be whether $s \beta^{2}$ is considered to be large or small, respectively.

Another alternative might be a power-law-like behaviour of the strengths, such as $\sigma_{p}^{2}=1 / p^{\alpha}$. Also in this case we may compute the $R_{k}$, as follows:

$$
R_{k}=\sum_{p>0} Q_{F}^{\Sigma}(p) \frac{1}{p^{k \alpha}}=\frac{1}{\Gamma(k \alpha)} \int_{0}^{\infty} z^{k \alpha-1}\left(F_{s}^{(3)}\left(e^{-z}\right)-1\right) d z,
$$

from which it follows that $\alpha>s$ to ensure convergence of $\mathrm{E}\left[D_{N}\right]$. In the large-s limit, we therefore find that, also in this case, $\gamma_{3} \rightarrow 1 / 2 s$.

\subsubsection{Fourier diaphony with spherical clustering}

A clustering choice which is, at least in principle, even more attractive from the symmetry point of view than sum clustering, is to let $\sigma_{n}$ depend on $|\boldsymbol{k}(n)|^{2}$, hence assuring the maximum possible amount of rotational invariance under the constraint of translational invariance. We therefore consider the choice

$$
\sigma_{n}^{2}=\exp \left(-\alpha \sum_{\mu} k\left(n_{\mu}\right)^{2}\right) .
$$

For the function $\beta\left(x_{1}, x_{2}\right)=\beta\left(x_{1}-x_{2}\right)$ we now have the foilowing two alternative forms, related by Poisson summation:

$$
\begin{aligned}
\beta(x) & =-1+\prod_{\mu=1}^{s}\left(\sum_{k=-\infty}^{+\infty} e^{-\alpha k^{2}} \cos \left(2 \pi k x^{\mu}\right)\right) \\
& =-1+\left(\frac{\pi}{\alpha}\right)^{s / 2} \sum_{m} \exp \left(-\frac{\pi^{2}(x+m)^{2}}{\alpha}\right) .
\end{aligned}
$$

of which the first converges well for large, and the second for small, values of $\alpha$; the sum over $m$ extends over the whole integer lattice. The $R_{k}$ are, similarly, given by

$$
\begin{aligned}
\kappa_{k} & =\left(\sum_{q=-\infty}^{+\infty} e^{-k \alpha q^{2}}\right)^{s}-1 \\
& =\left(\frac{\pi}{k \alpha}\right)^{s / 2}\left(\sum_{n=-\infty}^{+\infty} e^{-\pi^{2} m^{2} / k \alpha}\right)^{s}-1 .
\end{aligned}
$$

For large $\alpha$ (where, again, only the first few modes really contribute) we recover, again, the limit $\gamma_{3} \rightarrow 1 / 2 s$ as $s \rightarrow \infty$ : for small $\alpha$ we have, again, an exponential approach to the Gaussian limit,

$$
\gamma_{3} \sim\left(\frac{8 \alpha}{9 \pi}\right)^{s / 2} \text { as } s \rightarrow \infty
$$

The distinction between the two limiting behaviours is now the magnitude of the quantity $s \exp (-2 \alpha)$, which now takes over the rôle of the $s \beta^{2}$ of the previous paragraph. 


\subsubsection{Walsh diaphony}

Another type of diaphony is based on Walsh functions, which are defined as follows. Let, in one dimension, the real number $x$ be given by the decomposition

$$
x=2^{-1} x_{1}+2^{-2} x_{2}+2^{-3} x_{3}+\cdots, \quad x_{i} \in\{0,1\},
$$

and let the nonnegative integer $n$ be given by the decomposition

$$
n=n_{1}+2 n_{2}+2^{2} n_{3}+2^{3} n_{4}+\cdots, \quad n_{i} \in\{0,1\} .
$$

Then, the $n$th Walsh function $W_{n}(x)$ is defined as

$$
W .(x)=(-1)^{\left(n_{1} x_{1}+n_{2} x_{2}+n_{3} x_{3}+\cdots\right)} \text {. }
$$

The extension to the multidimensional case is of course straightforward, and it is easily seen that the Walsh functions form an orthonormal set. The Walsh diaphony is then given by

$$
D_{N}=\frac{1}{N} \sum_{n>0} \sigma_{n}^{2}\left(\sum_{k=1}^{N} W_{n}\left(x_{k}\right)\right)^{2}
$$

In Ref. [13], the following choice is made:

$$
\begin{aligned}
& \sigma_{n}^{2}=\frac{1}{3^{s}-1} \prod_{\mu=1}^{s} \frac{1}{r\left(n_{\mu}\right)^{2}}, \\
& r(n)=\theta(n=0)+\theta(n>0) \sum_{p \geq 0} 2^{p} \theta\left(2^{p} \leq n<2^{p+1}\right) .
\end{aligned}
$$

Note that, in contrast to the Fourier case where each mode of frequency $n$ contains two basis functions (one sine and one cosine), the natural requirement of 'translational invariance' in this case requires that the Walsh functions from $2^{p}$ up to $2^{p+1}$ get equal strength. The clusterings are therefore quite different from the Fourier case. We slightly generalize the notions of Ref. [13], and write

$$
\begin{aligned}
& \sigma_{n}^{2}=\prod_{\mu=1}^{s} \frac{1}{r\left(n_{\mu}\right)^{2}}, \\
& r(n)=\theta(n=0)+\theta(n>0) \sum_{p \geq 0}\left(\alpha \beta^{p}\right)^{-1 / 2} \theta\left(2^{p} \leq n<2^{p+1}\right) .
\end{aligned}
$$

Here, we have disregarded the overall normalization of the $\sigma$ 's since it does not influence the Gaussian limit. It is an easy matter to compute the $R_{k}$; we find

$$
R_{k}=\sum_{n>0} \sigma_{n}^{2 k}=\left(1+\frac{\alpha^{k}}{1-2 \beta^{k}}\right)^{s}-1,
$$

so that the requirement $\mathrm{E}\left[D_{N}\right]=R_{1}<\infty$ implies that we must have $\beta<1 / 2$. Therefore, for not too small values of $\alpha$, we have

$$
\gamma_{3} \sim a(\alpha, \beta)^{s}, \quad a(\alpha, \beta)=\frac{\left(1+\alpha^{3} /\left(1-2 \beta^{3}\right)\right)^{2}}{\left(1+\alpha^{2} /\left(1-2 \beta^{2}\right)\right)^{3}} .
$$

The choice made in Ref. [13] corresponds to $\alpha=1$ and $\beta=1 / 4$, for which we find $a(1,1 / 4) \sim 0.4197$. The Gaussian limit should, therefore, be a good approximation for $s$ larger than 6 or so. An interesting observation 
is that for fixed $\beta, a(\alpha, \beta)$ attains a minimum at $\alpha=\left(1-2 \beta^{3}\right) /\left(1-2 \beta^{2}\right)$, so that the choice $\beta=1 / 4$ could in principle lead to $a(31 / 28,1 / 4)=0.4165$ with a marginally faster approach to the Gaussian. The overall infimum is seen to be $a(3 / 2,1 / 2)=2 / 11 \sim 0.182$. As in the Fourier case with product clustering and a power-law strength, there is a limit on the speed with which the Gaussian is approached: in both cases this is directly related to the type of clustering.

At the other extreme, for very small $\alpha$ we find the limiting behaviour

$$
\gamma_{3} \sim \frac{\left(1-2 \beta^{2}\right)^{3}}{\left(1-2 \beta^{3}\right)^{2}} \frac{1}{s} \text { if } s \alpha^{2} \ll 1
$$

Again in this case, the slowest possible approach to the Gaussian limit is like $1 / s$, directly related to the symmetry of the discrepancy definition with respect to the various coordinate axes.

\subsection{Lego discrepancy}

Another class of integrands and discrepancies can be constructed by dissecting the hypercube $K$ into $M$ non-overlapping bins $A_{m}(m=1,2, \ldots, M)$, and taking the characteristic functions $\vartheta_{m}$ of the bins as the basis functions of the measure. Then $w_{n}$ is the volume of $A_{m}$, and

$$
\sum_{m=1}^{M} w_{n}=1 \text { and } a_{m, n}=w_{n} \delta_{n, n} .
$$

Note that in this case $n$ runs over a finite set of values. Moreover, this model is dimension-independent, in the sense that the only information on the dimension of $K$ is that contained in the value of $M$ : if the dissection of $K$ into bins $A_{k}$ is of the hyper-cubic type with $p$ bins along each axis, then we shall have $M=p^{s}$. Also, a general area-preserving mapping of $K$ onto itself, such as the Arnol'd cat-transform, will leave the discrepancy invariant: it will lead to a distortion (and possibly a dissection) of the various bins $A_{m}$, but this influences neither $w_{m}$ nor (by definition) $\sigma_{m}$. Owing to the finiteness of $M$, a finite point set can, in fact, have zero discrepancy in this case, namely if every bin $A_{m}$ contains precisely $w_{m} N$ points (assuming this number to be integer for every $m$ ). bins,

The matrix $\Gamma_{m, n}$ has now indices that label the bins $(m, n=1,2, \ldots M)$, where $M$ is the total number of

$$
\Gamma_{m, n}=\sigma_{m} \sigma_{n}\left(w_{m} \delta_{m, n}-w_{m} w_{n}\right) .
$$

We shall now examine under what circumstances the criterion (31) for the appearance of the Gaussian limit is fulfilled. The eigenvalues $\lambda_{i}$ of the matrix $\Gamma_{m, n}$ are, of course, given as the roots of the eigenvalue equation

$$
\left(\prod_{m=1}^{M}\left(\lambda_{i}-\sigma_{m}^{2} w_{m}\right)\right)\left(\sum_{n=1}^{M} \frac{w_{n} \lambda_{i}}{\lambda_{i}-\sigma_{n}^{2} w_{n}}\right)=0 .
$$

It is seen that there is always one zero eigenvalue (the corresponding eigenvector has $1 / \sigma_{m}$ for its $m$ th component). Furthermore, the eigenvalues are bounded by $\max _{m}\left(\sigma_{m}^{2} w_{m}\right)$, and this bound is an eigenvalue if there is more than one $m$ for which the maximum is attained. At any rate, we have for our criterion, that

$$
C=\frac{\lambda^{2}}{\sum_{i} \lambda_{i}^{2}} \leq \frac{\max _{m}\left(\sigma_{m}^{2} w_{m}\right)^{2}}{\operatorname{Tr}\left(\Gamma^{2}\right)}=\frac{\max _{m}\left(\sigma_{m l}^{2} w_{m}\right)^{2}}{\sum_{n t} \sigma_{m}^{4} w_{m}^{2}\left(1-2 w_{m}\right)+\left(\sum_{m} \sigma_{m}^{2} w_{m i}\right)^{2}} .
$$

Since the generality of the Lego discrepancy allows us to choose from a multitude of possibilities for the $\sigma$ 's and $w$ 's, we now concentrate on a few special cases. 
(1) All $w_{m}$ equal. This models integrands whose local details are not resolved within areas smaller than $1 / M$, but whose magnitude may fluctuate. In that case, we have

$$
C<\frac{1}{1-2 / M} \frac{\left(\max _{m} \sigma_{m}\right)^{4}}{\sum_{n} \sigma_{n}^{4}},
$$

and a sufficient condition for the Gaussian limit is for this bound to approach zero. Note that here, as in the general case, only bins $m$ with $\sigma_{m} \neq 0$ contribute to the discrepancy as well as to the criterion $C$, so that one has to be careful with models in which the integrand is fixed at zero in a large part of the integration region $K$ : this type of model was, for instance, examined in Ref. [14].

(2) $A l l \sigma_{m}$ equal. In this case, the underlying integrands have more or less bounded magnitude, but show finer detail in some places (with small $w$ ) than in other places (with larger $w$ ). Now, it is simple to prove that

$$
C \leq \frac{M \bar{w}^{2}}{1-2 \bar{w}+1 / M}, \quad \bar{w}=\max _{m} w_{m},
$$

so that a sufficient condition is that $M \bar{w}^{2}$ should approach zero.

(3) $A l l \sigma_{m}^{2} w_{m}$ equal. This choice models functions in which the largest fluctuations appear over the smallest intervals. Although not a priori attractive in many cases, this choice is actually quite appropriate for, e.g. particle physics where cross sections display precisely this kind of behaviour. In this case we simply have

$$
C=\frac{1}{(M+2)(M-1)},
$$

and the Gaussian limit follows whenever $M \rightarrow \infty$.

\section{Conclusions}

We have shown that a large class of discrepancies, including the $L_{2}$ star-discrepancy and the diaphonies, can be formulated as the induced discrepancy of a class of functions defined by a countable set of basis functions. These basis functions we called modes. For such a discrepancy we derived the probability distribution, in the limit of a large number of points, over the ensemble of truly random point-sets. We have shown under what conditions this distribution tends to a Gaussian. In particular, the question of the limiting behaviour of a given distribution can be reduced to solving an eigenvalue problem. Using the knowledge of the eigenvalues for a given function class it is possible to determine under which conditions and how fast the Gaussian linit is approached. Finally, we have investigated the limiting behaviour of the probability distribution for the discrepancy of several function classes explicitly.

The discrepancy that fastest approaches the Gaussian limit is obtained for the model in which the number of modes with nonzero equal strength goes to infinity, while the sum of the strengths is fixed. In fact, we give an argument why we cannot improve much on this limit. However, a drawback of this model is that the discrepancy itself becomes a sum of Dirac $\delta$-distributions in this limit: it only measures whether points coalesce, and is therefore not very useful in practice.

Secondly, we looked at the $L_{2}$ star-discrepancy. Here a Gaussian distribution appears in the limit of a large number of dimensions. It is, however, a very slow limit: only when the number of dimensions becomes of the order $\mathcal{O}\left(10^{2}\right)$ does the Gaussian behaviour become manifest.

For the different diaphonies the choice of the mode-strengths is more arbitrary. The strengths we discuss are chosen on the basis of some preferred global properties of the diaphony, such as translation-and/or rotationinvariance. Again for large dimensions the Gaussian limit is attained, either as a power-law or inverse of the 
number of dimension. It is possible to choose the strengths in such a way that the Gaussian limit is approached arbitrarily fast. But the diaphony corresponding to that case again consists of a sum of Dirac $\delta$-distributions.

Finally, for the Lego-discrepancy, we can assign strengths to the different modes in several ways. One example is to keep the product of the squared strength and volume of the modes fixed, then the Gaussian limit is reached for a large number of modes.

All these results have been derived in the limit of large number of points. It remains to be seen, however, whether this is reasonable in practice. To determine when the asymptotic regime sets in, i.e. for which value of $N$, it is necessary to take into account the next-to-leading contributions. This will be the subject of Ref. [15].

\section{Appendix A. The form of $G_{0}(z)$}

In this appendix we derive the result (24) for the form of $G_{0}(z)$ in terms of the quantities $A$ and $B$ of Eq. (23). For simplicity of notation, we shall assume the discrete case where the $A_{m, n}$ is a matrix, and the $B_{m}$ a vector; the indices $m, n$ are then what we called the variables $y$ in the foregoing. Moreover, let us denote by $\left[B A^{k} B\right]$ the sum $\sum_{m, n} B_{m}\left(A^{k}\right)_{m, n} B_{n}$. Since the matrix $\Gamma_{m, n}$ can be written as

$$
\Gamma_{m, n}=A_{m, n}-B_{m} B_{n},
$$

the $k$ th power of this matrix has the general form

$$
\left(\Gamma^{k}\right)_{m, n}=\left(A^{k}\right)_{m, n}-\sum_{p, q, \nu_{0.1,2} \geq 0} \frac{\left(\sum_{r \geq 0} \nu_{r}\right) !}{\nu_{0} ! \nu_{1} ! \nu_{2} ! \cdots}\left(A^{p} B\right)_{m}\left(B A^{q}\right)_{n} \prod_{r \geq 0}\left(-\left[B A^{r} B\right]\right)^{\nu_{r}},
$$

with the constraint $k-1=p+q+\nu_{0}+2 \nu_{1}+3 \nu_{2}+\cdots$. The combinatorial factor follows directly from the possible positionings of the dyadic factors $-B_{m} B_{n}$. Multiplying by $(2 t)^{k-1}$ and summing over the $k$ then gives us immediately

$$
\operatorname{Tr}\left(\frac{\Gamma}{1-2 t \Gamma}\right)=\sum_{k \geq 1}(2 t)^{k-1} \operatorname{Tr}\left(A^{k}\right)+\frac{-1}{1+\sum_{n \geq 1}(2 t)^{n}\left[B A^{n-1} B\right]} \sum_{r \geq 0}(r+1)(2 t)^{r}\left[B A^{r} B\right],
$$

where the last factor, with $r+1$, comes from the double sum over $p$ and $q$ with $p+q=r$. Upon integration of this result over $t$ from 0 to $z$, we find

$$
\begin{aligned}
\log \left(G_{0}(z)\right) & =\sum_{n>0} \frac{(2 z)^{n}}{2 n} \operatorname{Tr}\left(\Gamma^{n}\right) \\
& =\sum_{n>0} \frac{(2 z)^{n}}{2 n} \operatorname{Tr}\left(A^{n}\right)-\frac{1}{2} \log \left(1+\sum_{n>0}(2 z)^{n}\left[B A^{n-1} B\right]\right) .
\end{aligned}
$$

This result has, in fact, already been obtained for the case of the $L_{2}$ star-discrepancy in Ref. [5], but here we demonstrate its general validity for more general discrepancy measures. In those cases where $B_{m}=0$, the second term of course vanishes.

\section{Appendix B. A counterexample}

In this appendix we prove that the condition (31) for the occurrence of a Gaussian limit is, in a sense, the best possible. Namely, consider a set of eigenvalues $\lambda_{n}$, again adding up to unity as usual, defined as follows: 


$$
\begin{aligned}
& \lambda_{1}=\lambda, \\
& \lambda_{n}=(1-\lambda) /(M-1), \quad n=2,3, \ldots, M, \\
& \lambda_{n}=0, \quad n>M .
\end{aligned}
$$

Clearly, $\lambda$ will indeed be the maximal eigenvalue as long as $M>1 / \lambda$. Now,

$$
\frac{\lambda^{2}}{\sum_{n} \lambda_{n}^{2}}=\frac{\lambda^{2}}{\lambda^{2}+(1-\lambda)^{2} /(M-1)}
$$

and this ratio can be driven as close to unity as desired by choosing $M$ sufficiently large. This shows that the simple condition $\lambda \rightarrow 0$ is not always enough to ensure the Gaussian limit.

\section{Appendix C. Spectral representation of the $L_{2}$ star-discrepancy}

Mercer's theorem [17] states that a nonnegative-definite and continuous function on $(1,0] \times(1,0]$ has a spectral decomposition. Applying this to the function $\min \left(x_{1}^{\mu}, x_{2}^{\mu}\right)$ then tells us that the two-point connected Green's function $g$ of the Wiener measure has a spectral decomposition of Eq. (39). The eigenvalues $\sigma_{n}^{2}$ and eigenfunctions $u_{n}$ for $g$ are given by Eq. (41) and Eq. (40).

To show that the discrepancy defined through the functions $u_{n}$ is the same as the $L_{2}$ star-discrepancy pinned down at $(1,1, \ldots, 1)$, we prove the equality of the $\beta$-functions for the two measures,

$$
\begin{aligned}
& \sum_{n} \sigma_{n}^{2}\left(u_{n}\left(x_{1}\right)-2^{s / 2} \sigma_{n}\right)\left(u_{n}\left(x_{2}\right)-2^{s / 2} \sigma_{n}\right) \\
& =\int_{K^{\prime}}\left(\prod_{\mu=1}^{s} \theta\left(x_{1}^{\mu}-y^{\mu}\right)-\prod_{\mu=1}^{s}\left(1-y^{\mu}\right)\right)\left(\prod_{\mu=1}^{s} \theta\left(x_{2}^{\mu}-y^{\mu}\right)-\prod_{\mu=1}^{s}\left(1-y^{\mu}\right)\right) d y .
\end{aligned}
$$

Evaluating both sides of the equation, we obtain

$$
\begin{aligned}
& \sum_{n}\left(\sigma_{n}^{2} u_{n}\left(x_{1}\right) u_{n}\left(x_{2}\right)-2^{s / 2} \sigma_{n}^{3} u_{n}\left(x_{1}\right)-2^{s / 2} \sigma_{n}^{3} u_{n}\left(x_{2}\right)+2^{s} \sigma_{n}^{4}\right) \\
& =g\left(x_{1}, x_{2}\right)-\prod_{\mu=1}^{s}\left(x_{1}^{\mu}-\frac{1}{2}\left(x_{1}^{\mu}\right)^{2}\right)-\prod_{\mu=1}^{s}\left(x_{2}^{\mu}-\frac{1}{2}\left(x_{2}^{\mu}\right)^{2}\right)+\left(\frac{1}{3}\right)^{s} .
\end{aligned}
$$

The first terms on both sides of the equation cancel trivially. A small calculation shows that the same applies to the last terms on both sides of the equation. It thus remains to show that

$$
\sum_{n} 2^{s / 2} \sigma_{n}^{3} u_{n}(x)=\prod_{\mu=1}^{s}\left(x^{\mu}-\frac{1}{2}\left(x^{\mu}\right)^{2}\right) .
$$

This problem again factorizes for the different coordinates (omitting indices),

$$
2\left(\frac{2}{\pi}\right)^{3} \sum_{n=0}^{\infty} \frac{1}{(2 n+1)^{3}} \sin \left((2 n+1) \frac{\pi}{2} x\right)=x-\frac{1}{2} x^{2}
$$

which is nothing but stating that the l.h.s. of Eq. (C.4) is the Fourier decomposition of the r.h.s. To prove this, let $f$ be the following periodic extension of $x-\frac{1}{2} x^{2}$ : 


$$
f(x)= \begin{cases}\frac{1}{2} x^{2}-3 x+4, & x \in(2 m-2,2 m], \\ x-\frac{1}{2} x^{2}, & x \in(2 m, 2 m+2],\end{cases}
$$

where $m$ is any integer. The function $f$ is a parabolic approximation of $\frac{1}{2} \sin (4 \pi x)$. It is continuous and differentiable on $\mathbb{R}$. Hence it can be written as a Fourier series, based on a period of 4 rather than 1 . An explicit calculation shows that the only nonzero terms come from the functions $\frac{1}{\sqrt{2}} \sin \left((2 n+1) \frac{\pi}{2} x\right)(n=0,1,2, \ldots)$. The Fourier coefficients are given by ${ }^{7}$

$$
\frac{1}{\sqrt{2}} \int_{0}^{4} f(x) \sin \left((2 n+1) \frac{\pi}{2} x\right) d x=2 \sqrt{2}\left(\frac{2}{\pi}\right)^{3} \frac{1}{(2 n+1)^{3}}
$$

Thus the Fourier series is exactly given by the I.h.s. of Eq. (C.4).

\section{Appendix D. The magnitude of $Q_{F}^{\mathrm{n}}(p)$}

Here we present the proofs of our various statements about the multiplicity function $Q_{F}^{\text {II }}(p)$ of Section 3.3.3. In the first place, we know that its Dirichlet generating function, $F^{(1)}(x)$, converges for all $x>1$. Now suppose that $Q_{F}^{\Pi}(p)$ exceeded $c p^{\alpha}$ an infinite number of times, with $c>0$ and $\alpha>1$. The Dirichlet generating function would then contain an infinite number of terms all larger than $c$, for $1<x<\alpha$, and therefore would diverge, in contradiction with its convergence for all $x>1$.

In the second place, consider the 'standard' generating function, $F_{s}^{(2)}(x)$. By inspecting how many of the vector components $n_{\mu}$ of $n$ are zero, we see that we may write, for $p>1$,

$$
Q_{r}^{\Pi}(p)=\sum_{t=1}^{s}\left(\begin{array}{l}
s \\
t
\end{array}\right) 2^{t} d_{l}(p), \quad d_{t}(p)=\sum_{n \geq 0} \theta\left(p=\prod_{\mu=1}^{t} n_{\mu}\right),
$$

so that $d_{t}(p)$ counts in how many ways the integer $p$ can be written as a product of $t$ factors, including ones; this function is discussed, for instance, in Ref. [16]. Now, for $p$ prime, we have $d_{t}(p)=t$, and therefore

$$
Q_{F}^{\Pi}(p) \geq 2 s\left(3^{s-1}\right), \quad \text { equality for } p \text { prime. }
$$

The radius of convergence of $F_{s}^{(2)}(x)$ is therefore at most equal to unity. On the other hand, we can obtain a very crude, but sufficient, upper bound on $Q_{F}^{\Pi}(p)$ as follows. Since $d_{t}(p)$ is a nondecreasing function of $t$, we may bound $Q_{F}^{\Pi}(p)$ by $\left(3^{s}-1\right) d_{s}(p)$. Now let $k_{p}$ be the number of prime factors in $p$; then $k_{i}$ cannot exceed $\log (p) / \log (2)$, and only is equal to this when $p$ is a purc power of 2 . Also, the number of ways to distribute $k$ object in $s$ groups (which may be empty) is at most $s^{k}$, and is smaller if some of the objects are equal. Therefore, $d_{s}(p)$ is at most $s^{k_{p}}$, and we see that

$$
Q_{F}^{\mathrm{\Pi}}(p)<\left(3^{s}-1\right) p^{\log (s) / \log (2)},
$$

or, in short, is bounded ${ }^{8}$ by a polynomial in $p$. Therefore, the radius of convergence of $F_{s}^{(2)}(x)$ is also at least unity, and we have proven the assertion in Eq. (3.3.3).

\footnotetext{
${ }^{7}$ We take the functions normalized such that they form a orthonornal set on $(0,4)$, so the Fourier series is in terms of the sine- and cosine functions divided by $\sqrt{2}$.

${ }^{8}$ Note that equality cannot occur in this case since the two requirements are mutually exclusive.
} 
Finally, we consider the limit

$$
\lim _{\beta^{\prime} \rightarrow 1} \gamma_{3}=\lim _{x \rightarrow 1} \frac{\left(F_{s}^{(2)}\left(x^{3}\right)\right)^{2}}{\left(F_{s}^{(2)}\left(x^{2}\right)\right)^{3}} .
$$

The same reasoning that led us to the radius of convergence shows that, for $x$ approaching 1 from below, the function $F_{s}^{(2)}(x)$ behaves as $(1-x)^{-c}$, with $c \geq 1$. Therefore, $\gamma_{3}$ will behave as $(8(1-x) / 9)^{c}$, and approach zero as $x \rightarrow 1$. Note that the upper bound on $Q_{F}^{\Pi}(p)$ is extremely loose: but it is enough.

\section{References}

[1] L. Kuipers, H. Niederreiter, Uniform Distribution of Sequences (Wiley, New York, 1974) R.F. Tichy, M. Drmota, Sequences, Discrepancies and Applications (Springer, Berlin, 1997).

[2] H. Niederreiter, Random Number Generation and Quasi-Monte Carlo Methods (SIAM, Philadelphia, PA, 1992).

[3] P. Zinterhof, Über einige Abschätzungen bei der Approximation von Funktionen met Gleichverteilungsmethoden, Sitzungsber. Österr. Akad. Wiss. Math.-Natur. Kl.II 185 (1976) 121-132:

P. Hellekalek, Correlations between pseudorandom numbers: theory and numerical practice, Proc. 1st Salzburg Minisymposium on Pseudorandom Number Generation and Quasi-Monte Carlo Methods, P. Hellekalek, G. Larcher, P. Zinterhof, eds. (Salzburg, 1994).

[4] R. Kleiss, Average-case complexity distributions: a generalization of the Woźniakowski lemma for multidimensional numerical integration, Comput. Phys. Commun. 71 (1992) 39-53.

[5] F. James, J. Hooglend, R. Kleiss, Multidimensional sampling for simulation and integration: measures, discrepancies and quasi-random numbers, Comput. Phys. Commun. 99 (1997) 180-220.

[6] H. Woźniakoski, Average-case complexity of multivariate integration, Bull. AMS 24 (1991) 185-194.

[7] S. Paskov, Average-case complexity of multivariate integration for smooth functions, J. Complexity 9 (1993) $291-312$.

[8] S. Tezuka, Polynomial arithmetic analogue of Halton sequences, ACM Trans. Modeling \& Computer Simulation 3 (1993) 99-107; S. Ninomiya, S. Tezuka, Thward real-time pricing of complex financial derivatives, Appl. Math. Finance 3 (1996) 1-20.

[9] I.K. Hoogland, Radiative corrections, Quasi-Monte Carlo and Discrepancy, Ph.D. thesis (University of Amsterdam, Amsterdam, 1996).

[10] J. Hoogland, R. Kleiss. Discrepancy-based error estimates for Quasi-Monte Carlo. I. General formalism. Comput. Phys. Commun. 98 (1996) 111-127.

[11] J. Hoogland, R. Kleiss, Discrepancy-based error estimates for Quasi-Monte Carlo. II. Results for one dimension, Comput. Phys. Commun. 98 (1996) 128-136.

[12] J. Hoogland, R. Kleiss, Discrepancy-based error estimates for Quasi-Monte Carlo. III. Error distributions and central limits, Comput. Phys. Commun. 101 (1997) 21-30.

[13] H. Leeb, Weak limits for diaphony, Proc. 2nd Int. Conference on Monte Carlo and Quasi-Monte Carlo Methods in Scientific Computing, H. Niederreiter, P. Hellekalek, G. Larcher, P. Zinterhof, eds., Lecture Notes in Statistics (Springer, Berlin), to appear.

[14] M. Berblinger, Ch. Schlier, T. Weiss, Monte Carlo integration with quasi-random numbers: experience with discontinuous integrands, Comput. Phys. Commun. 99 (1997) 151-162.

[15] A. van Hameren, J. Hoogland, R. Kleiss, Gaussian limits for discrepancies. II. Sub-leading corrections and the curse of dimensionality. in preparation.

[16] G.H. Hardy, E.M. Wright, An Introduction to the Theory of Numbers (Oxford, 1988).

[17] M. Lòve, Probability Theory, 3rd ed. (Van Nostrand, Princeton, NJ, 1963). 